International Mathematical Forum, 2, 2007, no. 63, 3143 - 3153

\title{
Some Classes of Analytic Functions Involving Generalized Integral Operator
}

\author{
C. Selvaraj \\ Department of Mathematics, Govt. Arts College (Men) \\ Nandanam, Chennai-600 035 \\ Tamilnadu, India \\ K. R. Karthikeyan \\ Department of Mathematics, R. M. K. Engineering College \\ R. S. M. Nagar, Kavaraipettai-601206 \\ Tamilnadu, India \\ kr_karthikeyan1979@yahoo.com
}

\begin{abstract}
The purpose of the present article is to introduce several new subclasses of analytic functions using certain integral operator and investigate various inclusion relations for these subclasses. Some interesting applications involving a certain class of integral operator are also considered.
\end{abstract}

Mathematics Subject Classification: 30C45, 30C50, 30C75

Keywords: univalent function; convex function; starlike function; closeto-convex function; quasi-convex function; integral operators; differential subordination

\section{Introduction, Definitions And Preliminaries}

Let $\mathcal{A}$ denote the class of analytic functions in the unit disc $\mathcal{U}$ with the normalization $f(0)=f^{\prime}(0)-1=0$. Also let $\mathcal{S}^{*}(\alpha), \mathcal{K}(\alpha), \mathcal{C C}(\alpha, \beta)$ and $\mathcal{C}^{*}(\alpha, \beta)$ denote the subclasses of $\mathcal{A}$ consisting of functions which are starlike of order $\alpha$, convex of order $\alpha$, close-to convex of order $\alpha$ type $\beta$ and quasi-convex of order $\alpha$ type $\beta$ in $\mathcal{U}$ respectively. For details regarding these subclasses and various other classes we refer to $[1,2,8]$.

Let $\mathcal{N}$ be the class of all functions $\phi$ which are analytic and univalent in $\mathcal{U}$ and for which $\phi(\mathcal{U})$ is convex with $\phi(0)=1$ and $\operatorname{Re}\{\phi(z)\}>0, z \in \mathcal{U}$. 
Making use of the principle of subordination between analytic functions, several authors have investigated the subclasses $\mathcal{S}^{*}(\alpha, \phi)$ and $\mathcal{K}(\alpha, \phi)$ of the class $\mathcal{A}$ for $\phi, \psi \in \mathcal{N}$ which are defined as follows:

$$
\begin{gathered}
\mathcal{S}^{*}(\alpha, \phi):=\left\{f: f \in \mathcal{A} \text { and } \frac{1}{1-\alpha}\left(\frac{z f^{\prime}(z)}{f(z)}-\alpha\right) \prec \phi(z),(z \in \mathcal{U})\right\}, \\
\mathcal{K}(\alpha, \phi):=\left\{f: f \in \mathcal{A} \text { and } \frac{1}{1-\alpha}\left(1+\frac{z f^{\prime \prime}(z)}{f^{\prime}(z)}-\alpha\right) \prec \phi(z),(z \in \mathcal{U})\right\}
\end{gathered}
$$

respectively. We now introduce the following:

$$
\begin{gathered}
\mathcal{C}^{\prime}(\alpha, \beta: \phi, \psi):=\{f: f \in \mathcal{A} \text { and } \exists g \in \mathcal{K}(\alpha, \phi) \text { s.t. } \\
\left.\frac{1}{1-\beta}\left(\frac{z f^{\prime}(z)}{g(z)}-\beta\right) \prec \psi(z),(z \in \mathcal{U})\right\} \\
\mathcal{U}^{*}(\alpha, \beta: \phi, \psi):=\{f: f \in \mathcal{A} \text { and } \exists g \in \mathcal{K}(\alpha, \phi) \text { s.t. } \\
\left.\frac{1}{1-\beta}\left(\frac{z\left(z f^{\prime}(z)\right)^{\prime}}{g(z)}-\beta\right) \prec \psi(z),(z \in \mathcal{U})\right\} .
\end{gathered}
$$

Here we note that $0 \leq \alpha, \beta<1$ and the symbol $\prec$ denotes subordination.

Also it can be verified that for the choice of $\phi(z)=\psi(z)=\frac{1+z}{1-z}$, $\mathcal{C}^{\prime}(\alpha, \beta: \phi, \psi)=C^{\prime}(\alpha, \beta)$, a subclass of close-to-convex function introduced and studied by Selvaraj [11].

For two functions $f, g$ of the form

$$
f(z)=\sum_{n=0}^{\infty} a_{n} z^{n}, \quad g(z)=\sum_{n=0}^{\infty} b_{n} z^{n},
$$

their Hadamard product (or convolution) is defined, as usual, by

$$
(f * g)(z):=\sum_{n=0}^{\infty} a_{n} b_{n} z^{n}, \quad z \in \mathcal{U} .
$$


Let $f \in \mathcal{A}$. Denote by $D^{n}: \mathcal{A} \rightarrow \mathcal{A}$ the operator defined by

$$
D^{n} f=\frac{z}{(1-z)^{n+1}} * f(z), \quad(n>-1) .
$$

It is obvious that $D^{0} f=f(z), D^{1} f=z f^{\prime}(z)$ and $D^{n}=\frac{z\left(z^{n-1} f(z)\right)^{(n)}}{n !}$, $n \in \mathcal{N}_{0}=\{1,2, \ldots\}$. The operator $D^{n} f$ is called the nth order Ruscheweyh derivative of $f$. Analogous to $D^{n} f$, Noor [9] (see also Noor and Noor[10]) defined and studied an integral operator $I_{n}: \mathcal{A} \rightarrow \mathcal{A}$ as follows :

Let $f_{n}(z)=\frac{z}{(1-z)^{n+1}}, n \in N_{0}$ and let $f_{n}^{-1}(z)$ be defined such that

$$
f_{n}(z) * f_{n}^{-1}(z)=\frac{z}{(1-z)^{2}}, \quad(n>-1) .
$$

Then

$$
I_{n} f(z)=f_{n}^{-1}(z) * f(z)=\left[\frac{z}{(1-z)^{n+1}}\right]^{-1} * f(z) .
$$

The operator $I_{n}$ is called the Noor Integral operator of nth order of $f$. Define the familiar Gaussian hypergeometric function ${ }_{2} F_{1}(a, b, c ; z)$ by

$$
{ }_{2} F_{1}(a, b, c ; z):=\sum_{n=0}^{\infty} \frac{(a)_{n}(b)_{n}}{(c)_{n}} \frac{z^{n}}{n !}, \quad(a, b>0 ; c \neq 0,-1,-2, \ldots: z \in \mathcal{U})
$$

where $(x)_{k}$ is the Pochhammer symbol defined by

$$
(x)_{k}= \begin{cases}1 & \text { if } k=0 \\ x(x+1)(x+2) \ldots(x+k-1) & \text { if } k \in N_{0}=\{1,2, \ldots\} .\end{cases}
$$

Now we introduce an integral operator $I_{\lambda}^{p}(a, b, c): \mathcal{A} \longrightarrow \mathcal{A}$ as follows : Let $F_{\lambda, p}(z)=z+\sum_{n=2}^{\infty} \frac{(n+\lambda)^{p}}{(\lambda+1)^{p}} z^{n}, p \in N_{0}, \lambda \neq-1$ and let $F_{\lambda, p}^{-1}(z)$ be defined such that

$$
F_{\lambda, p}(z) * F_{\lambda, p}^{-1}(z)=z_{2} F_{1}(a, b, c ; z)
$$

Then,

$$
I_{\lambda}^{p}(a, b, c) f=F_{\lambda, p}^{-1}(z) * f(z) .
$$

We note that $I_{1}^{p}(1, b, b) f=J^{p} f$ an operator introduced by Jung,Kim and Srivastava [5] and also contains various other operators for the special choices 
of the parameters $\lambda, p, a, b, c$.

It can be easily verified from the above definition of the operator $I_{\lambda}^{p}(a, b, c) f$ that

$$
z\left(I_{\lambda}^{p+1}(a, b, c) f(z)\right)^{\prime}=(\lambda+1) I_{\lambda}^{p}(a, b, c) f(z)-\lambda I_{\lambda}^{p+1}(a, b, c) f(z)
$$

and

$$
z\left(I_{\lambda}^{p}(a, b, c) f(z)\right)^{\prime}=a I_{\lambda}^{p}(a+1, b, c) f(z)-(a-1) I_{\lambda}^{p}(a, b, c) f(z) .
$$

By using the integral operator $I_{\lambda}^{p}(a, b, c) f$, we introduce the following subclasses of analytic functions :

$$
\begin{gathered}
\mathcal{S}_{\lambda, p}^{*}(\alpha: a, b, c: \phi):=\left\{f: f \in \mathcal{A} \text { and } I_{\lambda}^{p}(a, b, c) f \in \mathcal{S}^{*}(\alpha, \phi)\right\} \\
\mathcal{K}_{\lambda, p}(\alpha: a, b, c: \phi):=\left\{f: f \in \mathcal{A} \text { and } I_{\lambda}^{p}(a, b, c) f \in \mathcal{K}(\alpha, \phi)\right\} \\
\mathcal{C}_{\lambda, p}^{\prime}(\alpha, \beta ; a, b, c ; \phi, \psi):=\left\{f: f \in \mathcal{A} \text { and } I_{\lambda}^{p}(a, b, c) f \in \mathcal{C}^{\prime}(\alpha, \beta: \phi, \psi)\right\}
\end{gathered}
$$

and

$$
\mathcal{U}_{\lambda, p}^{*}(\alpha, \beta ; a, b, c ; \phi, \psi):=\left\{f: f \in \mathcal{A} \text { and } I_{\lambda}^{p}(a, b, c) f \in \mathcal{U}^{*}(\alpha, \beta: \phi, \psi)\right\} .
$$

We note that

$$
f(z) \in \mathcal{K}_{\lambda, p}(\alpha: a, b, c: \phi) \quad \Longleftrightarrow z f^{\prime}(z) \in \mathcal{S}_{\lambda, p}^{*}(\alpha: a, b, c: \phi)
$$

and a similar relationship exists between the classes $\mathcal{C}_{\lambda, p}^{\prime}(\alpha, \beta ; a, b, c ; \phi, \psi)$ and $\mathcal{U}_{\lambda, p}^{*}(\alpha, \beta ; a, b, c ; \phi, \psi)$.

In this paper, we shall establish inclusion relation for these classes and some applications involving integral operators are also considered.

We now state the following lemmas which we may need to establish our results in the sequel.

Lemma 1.1 [6] Let $\beta, \gamma \in \mathcal{C}, \phi$ be convex univalent in $\mathcal{U}$ with $\phi(0)=1$ and $\operatorname{Re}(\beta \phi(z)+\gamma)>0, z \in \mathcal{U}$ and let $p(z)=1+p_{1} z+p_{2} z^{2}+\cdots$ be analytic in $\mathcal{U}$. Then

$$
p(z)+\frac{z p^{\prime}(z)}{\beta p(z)+\gamma} \prec \phi(z) \Rightarrow p(z) \prec \phi(z) \quad(z \in \mathcal{U})
$$

Lemma 1.2 [7] Let $\beta, \gamma \in \mathcal{C}, \phi$ be convex univalent in $\mathcal{U}$ and $w$ be analytic in $\mathcal{U}$ with $\operatorname{Re}\{w(z)\} \geq 0$. If $p$ is analytic in $\mathcal{U}$ and $p(0)=\phi(0)$, then the subordination:

$$
p(z)+w(z) z p^{\prime}(z) \prec \phi(z) \Longrightarrow p(z) \prec \phi(z)(z \in \mathcal{U}) .
$$




\section{Inclusion Properties Involving the Operator $I_{\lambda}^{p}(a, b, c) f$}

We begin with the following

Theorem 2.1 Let $\phi \in \mathcal{N}$ with $\max _{z \in \mathcal{U}} \operatorname{Re}\{\phi(z)\}<\min \left(\frac{\alpha+a-1}{\alpha-1}, \frac{\alpha+\lambda}{\alpha-1}\right)$ $(p, a, b, c>0 ; 0 \leq \alpha<1)$. Then

$$
\mathcal{S}_{\lambda, p}^{*}(\alpha: a+1, b, c: \phi) \subset \mathcal{S}_{\lambda, p}^{*}(\alpha: a, b, c: \phi) \subset \mathcal{S}_{\lambda, p+1}^{*}(\alpha: a, b, c: \phi) .
$$

Proof. To prove the first part of Theorem 2.1, let $f \in \mathcal{S}_{\lambda, p}^{*}(\alpha: a+1, b, c: \phi)$ and set

$$
p(z)=\frac{1}{1-\alpha}\left(\frac{z\left(I_{\lambda}^{p}(a, b, c) f(z)\right)^{\prime}}{I_{\lambda}^{p}(a, b, c) f(z)}-\alpha\right)
$$

where $p(z)=1+c_{1} z+c_{2} z^{2}+\ldots$ is analytic in $\mathcal{U}$ and $p(z) \neq 0$ for all $z \in \mathcal{U}$. Applying (8) in (10), we obtain

$$
a \frac{I_{\lambda}^{p}(a+1, b, c) f(z)}{I_{\lambda}^{p}(a, b, c) f(z)}=(1-\alpha) p(z)+\alpha+a-1 .
$$

By using the logarithmic differentiation on both side of (11), we have

$$
\frac{1}{1-\alpha}\left(\frac{z\left(I_{\lambda}^{p}(a+1, b, c) f(z)\right)^{\prime}}{I_{\lambda}^{p}(a+1, b, c) f(z)}-\alpha\right)=p(z)+\frac{z p^{\prime}(z)}{(1-\alpha) p(z)+\alpha+a-1} .
$$

Since

$$
\max _{z \in \mathcal{U}}(\operatorname{Re}\{\phi(z)\})<\frac{\alpha+a-1}{\alpha-1} \quad(z \in \mathcal{U} ; a>0 ; 0 \leq \alpha<1) .
$$

We see that $\operatorname{Re}\{(1-\alpha) \phi(z)+\alpha+a-1\}>0, \quad(z \in \mathcal{U})$.

Applying Lemma (1.1) to equation (12), it follows that $p(z) \prec \phi(z)$ in $\mathcal{U}$, that is

$$
f \in \mathcal{S}_{\lambda, p}^{*}(\alpha: a, b, c: \phi) .
$$

To prove the second inclusion relationship asserted by the Theorem (2.1), let

$$
f \in \mathcal{S}_{\lambda, p}^{*}(\alpha: a, b, c: \phi)
$$

and put

$$
s(z)=\frac{1}{1-\alpha}\left(\frac{z\left(I_{\lambda}^{p+1}(a, b, c) f(z)\right)^{\prime}}{I_{\lambda}^{p+1}(a, b, c) f(z)}-\alpha\right)
$$


where the function $s(z)$ is analytic in $\mathcal{U}$ with $s(0)=1$. Then, by using arguments similar to those detailed above with $(7)$, it follows that $s(z) \prec \phi(z)$ in $\mathcal{U}$, which implies that

$$
f \in \mathcal{S}_{\lambda, p+1}^{*}(\alpha: a, b, c: \phi)
$$

which completes the proof of the Theorem (2.1).

Remark 2.2 By taking $\lambda=1, a=1, b=c$ and $\phi(z)=\frac{1+z}{1-z}(z \in \mathcal{U})$ in Theorem (2.1), we obtain $\mathcal{S}_{p}^{*}(\alpha) \subset \mathcal{S}_{p+1}^{*}(\alpha)$, which was asserted earlier by Jin-Lin-Liu [4].

Theorem 2.3 Let $\phi \in \mathcal{N}$ with $\max _{z \in \mathcal{U}} \operatorname{Re}\{\phi(z)\}<\min \left(\frac{\alpha+a-1}{\alpha-1}, \frac{\alpha+\lambda}{\alpha-1}\right)$ $(p, a, b, c>0 ; 0 \leq \alpha<1)$. Then

$$
\mathcal{K}_{\lambda, p}(\alpha: a+1, b, c: \phi) \subset \mathcal{K}_{\lambda, p}(\alpha: a, b, c: \phi) \subset \mathcal{K}_{\lambda, p+1}(\alpha: a, b, c: \phi) .
$$

Proof. Applying (9) and Theorem (2.1), we observe that

$$
\begin{aligned}
f(z) \in \mathcal{K}_{\lambda, p}(\alpha: a+1, b, c: \phi) & \Longleftrightarrow I_{\lambda}^{p}(a+1, b, c) f(z) \in \mathcal{K}(\alpha, \phi) \\
& \Longleftrightarrow z\left(I_{\lambda}^{p}(a+1, b, c) f(z)\right)^{\prime} \in \mathcal{S}^{*}(\alpha, \phi) \\
& \Longleftrightarrow I_{\lambda}^{p}(a+1, b, c)\left(z f^{\prime}(z)\right) \in \mathcal{S}^{*}(\alpha, \phi) \\
& \Longleftrightarrow z f^{\prime}(z) \in \mathcal{S}_{\lambda, p}^{*}(\alpha: a+1, b, c: \phi) \\
& \Longleftrightarrow z f^{\prime}(z) \in \mathcal{S}_{\lambda, p}^{*}(\alpha: a, b, c: \phi) \\
& \Longleftrightarrow I_{\lambda}^{p}(a, b, c)(z f(z))^{\prime} \in \mathcal{S}^{*}(\alpha, \phi) \\
& \Longleftrightarrow z\left(I_{\lambda}^{p}(a, b, c) f(z)\right)^{\prime} \in \mathcal{S}^{*}(\alpha, \phi) \\
& \Longleftrightarrow I_{\lambda}^{p}(a, b, c) f(z) \in \mathcal{K}(\alpha, \phi) \\
& \Longleftrightarrow f(z) \in \mathcal{K}_{\lambda, p}(\alpha: a, b, c: \phi)
\end{aligned}
$$

and

$$
\begin{aligned}
f(z) \in \mathcal{K}_{\lambda, p}(\alpha: a, b, c: \phi) & \Longleftrightarrow z f^{\prime}(z) \in \mathcal{S}_{\lambda, p}^{*}(\alpha: a, b, c: \phi) \\
& \Longleftrightarrow z f^{\prime}(z) \in \mathcal{S}_{\lambda, p+1}^{*}(\alpha: a, b, c: \phi) \\
& \Longleftrightarrow z\left(I_{\lambda}^{p+1}(a, b, c) f(z)\right)^{\prime} \in \mathcal{S}^{*}(\alpha, \phi) \\
& \Longleftrightarrow f(z) \in \mathcal{K}_{\lambda, p+1}(\alpha: a, b, c: \phi)
\end{aligned}
$$

which evidently proves Theorem (2.3).

Taking $\phi(z)=\frac{1+A z}{1+B z} \quad(-1 \leq B<A \leq 1 ; z \in \mathcal{U})$ in Theorems (2.1) and (2.3), we have 
Corollary 2.4 Suppose that $\frac{1+A}{1+B}<\min \left(\frac{\alpha+a-1}{\alpha-1}, \frac{\alpha+\lambda}{\alpha-1}\right),(a, b, c ; p>0$, $\lambda>-1)$. Then

$$
\mathcal{S}_{\lambda, p}^{*}(\alpha: a+1, b, c: A, B) \subset \mathcal{S}_{\lambda, p}^{*}(\alpha: a, b, c: A, B) \subset \mathcal{S}_{\lambda, p+1}^{*}(\alpha: a, b, c: A, B)
$$

and

$\mathcal{K}_{\lambda, p}(\alpha: a+1, b, c: A, B) \subset \mathcal{K}_{\lambda, p}(\alpha: a, b, c: A, B) \subset \mathcal{K}_{\lambda, p+1}(\alpha: a, b, c: A, B)$.

Theorem 2.5 Let $\phi, \psi \in \mathcal{N}$ with $\max _{z \in \mathcal{U}} \operatorname{Re}\{\phi(z)\}<\min \left(\frac{\sigma+a-1}{\sigma-1}, \frac{\sigma+\lambda}{\sigma-1}\right)$. Then

$$
\mathcal{C}_{\lambda, p}^{\prime}(\alpha, \beta ; a+1, b, c ; \phi, \psi) \subset \mathcal{C}_{\lambda, p}^{\prime}(\alpha, \beta ; a, b, c ; \phi, \psi) \subset \mathcal{C}_{\lambda, p+1}^{\prime}(\alpha, \beta ; a, b, c ; \phi, \psi)
$$

Proof. We begin by proving that

$$
\mathcal{C}_{\lambda, p}^{\prime}(\alpha, \beta ; a+1, b, c ; \phi, \psi) \subset \mathcal{C}_{\lambda, p}^{\prime}(\alpha, \beta ; a, b, c ; \phi, \psi)
$$

Let $f(z) \in \mathcal{C}_{\lambda, p}^{\prime}(\alpha, \beta ; a+1, b, c ; \phi, \psi)$. Then, in view of the definition of the function class $\mathcal{C}_{\lambda, p}^{\prime}(\alpha, \beta ; a+1, b, c ; \phi, \psi)$, there exists a function $q \in \mathcal{K}(\alpha, \phi)$ such that

$$
\frac{1}{1-\beta}\left(\frac{z\left(I_{\lambda}^{p}(a+1, b, c) f(z)\right)^{\prime}}{q(z)}-\beta\right) \prec \psi(z) .
$$

Choose the function $g(z)$ such that $q(z)=I_{\lambda}^{p}(a+1, b, c) g(z)$, Then

$$
g \in \mathcal{K}_{\lambda, p}(\alpha: a+1, b, c: \phi) \text { and } \frac{1}{1-\beta}\left(\frac{z\left(I_{\lambda}^{p}(a+1, b, c) f(z)\right)^{\prime}}{I_{\lambda}^{p}(a+1, b, c) g(z)}-\beta\right) \prec \psi(z) \text {. }
$$

Now let,

$$
p(z)=\frac{1}{1-\beta}\left(\frac{z\left(I_{\lambda}^{p}(a, b, c) f(z)\right)^{\prime}}{I_{\lambda}^{p}(a, b, c) g(z)}-\beta\right)
$$

where the function $p(z)$ is analytic in $\mathcal{U}$ with $p(0)=1$. Using equation (8), we find that

$$
\begin{gathered}
\frac{1}{1-\beta}\left(\frac{z\left(I_{\lambda}^{p}(a+1, b, c) f(z)\right)^{\prime}}{I_{\lambda}^{p}(a+1, b, c) g(z)}-\beta\right)=\frac{1}{1-\beta}\left(\frac{\left(I_{\lambda}^{p}(a+1, b, c)\left(z f^{\prime}(z)\right)\right)}{I_{\lambda}^{p}(a+1, b, c) g(z)}-\beta\right) \\
\quad=\frac{1}{1-\beta}\left(\frac{z\left[I_{\lambda}^{p}(a, b, c)\left(z f^{\prime}(z)\right)\right]^{\prime}+(a-1) I_{\lambda}^{p}(a, b, c)\left(z f^{\prime}(z)\right)}{z\left(I_{\lambda}^{p}(a, b, c) g(z)\right)^{\prime}+(a-1) I_{\lambda}^{p}(a, b, c) g(z)}-\beta\right) \\
=\frac{1}{1-\beta}\left(\frac{\frac{z\left[I_{\lambda}^{p}(a, b, c)\left(z f^{\prime}(z)\right)\right]^{\prime}}{I_{\lambda}^{p}(a, b, c) g(z)}+(a-1) \frac{I_{\lambda}^{p}(a, b, c)\left(z f^{\prime}(z)\right)}{I_{\lambda}^{p}(a, b, c) g(z)}}{\frac{z\left(I_{\lambda}^{p}(a, b, c) g(z)\right)^{\prime}}{I_{\lambda}^{p}(a, b, c) g(z)}+(a-1)}-\beta\right) .
\end{gathered}
$$


Since $g \in \mathcal{K}_{\lambda, p}(\alpha: a+1, b, c: \phi) \subset \mathcal{K}_{\lambda, p}(\alpha: a, b, c: \phi)$ and using the fact that every convex function is starlike of order $1 / 2$ (see also jack[3]), we can set for some $0 \leq \sigma<1$

$$
r(z)=\frac{1}{1-\sigma}\left(\frac{z\left(I_{\lambda}^{p}(a, b, c) g(z)\right)^{\prime}}{I_{\lambda}^{p}(a, b, c) g(z)}-\sigma\right)
$$

where $r(z) \prec \phi(z)$ in $\mathcal{U}$ with the assumption that $\phi \in \mathcal{N}$. From (13), we have

$$
z\left[I_{\lambda}^{p}(a, b, c)\left(z f^{\prime}(z)\right)\right]^{\prime}=(1-\beta) p(z) z\left(I_{\lambda}^{p}(a, b, c) g(z)\right)+\beta z\left(I_{\lambda}^{p}(a, b, c) g(z)\right)
$$

Upon differentiating both sides of equation (15), we have

$$
\frac{z\left[I_{\lambda}^{p}(a, b, c)\left(z f^{\prime}(z)\right)\right]^{\prime}}{\left(I_{\lambda}^{p}(a, b, c) g(z)\right.}=(1-\beta) z p^{\prime}(z)+[(1-\beta) p(z)+\beta][(1-\sigma) q(z)+\sigma] .
$$

Then, by the virtue of the equations (13),(14) and (15), we have

$$
\frac{1}{1-\beta}\left(\frac{z\left(I_{\lambda}^{p}(a+1, b, c) f(z)\right)^{\prime}}{I_{\lambda}^{p}(a+1, b, c) g(z)}-\beta\right)=p(z)+\frac{z p^{\prime}(z)}{(1-\sigma) r(z)+\sigma+(a-1)} \prec \psi(z) .
$$

Since $a>0$ and $r(z) \prec \phi(z)$ in $\mathcal{U}$ with $\max _{z \in \mathcal{U}}(\operatorname{Re}\{\phi(z)\})<\frac{\sigma+a-1}{\sigma-1}$, we have

$$
\operatorname{Re}\{\sigma+a-1+(1-\sigma) r(z)\}>0 \quad(z \in \mathcal{U}) .
$$

Hence, by taking $w(z)=\frac{1}{\sigma+a-1+(1-\sigma) r(z)}$ in equation (17) and then applying Lemma (1.2), we can show that $p(z) \prec \psi(z)$ in $\mathcal{U}$, so that $f \in$ $\mathcal{C}_{\lambda, p}^{\prime}(\alpha, \beta ; a, b, c ; \phi, \psi)$.

For the second inclusion relationship asserted by the Theorem 2.5, using arguments similar to those detailed above with equation (7), we obtain

$$
\mathcal{C}_{\lambda, p}^{\prime}(\alpha, \beta ; a, b, c ; \phi, \psi) \subset \mathcal{C}_{\lambda, p+1}^{\prime}(\alpha, \beta ; a, b, c ; \phi, \psi)
$$

Thus, we have completed the proof of the Theorem(2.5).

Using the same method as in Theorem(2.3), we have the following:

Theorem 2.6 Let $\phi, \psi \in \mathcal{N}$ with $\max _{z \in \mathcal{U}} \operatorname{Re}\{\phi(z)\}<\min \left(\frac{\sigma+a-1}{\sigma-1}, \frac{\sigma+\lambda}{\sigma-1}\right)$. Then

$$
\mathcal{U}_{\lambda, p}^{*}(\alpha, \beta ; a+1, b, c ; \phi, \psi) \subset \mathcal{U}_{\lambda, p}^{*}(\alpha, \beta ; a, b, c ; \phi, \psi) \subset \mathcal{U}_{\lambda, p+1}^{*}(\alpha, \beta ; a, b, c ; \phi, \psi) .
$$




\section{Inclusion Properties Involving the Operator $L_{\mu}$}

In this section, we examine the closure properties of the integral operator $L_{\mu}(f)$ defined by

$$
L_{\mu}(f)=\frac{\mu+1}{z^{\mu}} \int_{0}^{z} t^{\mu-1} f(t) d t,(f \in \mathcal{A}, \mu>-1) .
$$

We first state and prove the following inclusion relationship for the integral operator $L_{\mu}(f)$.

Theorem 3.1 Let $\phi \in \mathcal{N}$ with $\max _{z \in \mathcal{U}} \operatorname{Re}\{\phi(z)\}<\frac{\alpha+\mu}{\alpha-1}(c>-1 ; 0 \leq \alpha<1)$. If $f \in \mathcal{S}_{\lambda, p}^{*}(\alpha: a, b, c: \phi)$, so is $L_{\mu}(f)$

Proof. Let $f \in \mathcal{S}_{\lambda, p}^{*}(\alpha: a, b, c: \phi)$ and set

$$
p(z)=\frac{1}{1-\alpha}\left(\frac{z\left(I_{\lambda}^{p}(a, b, c) L_{\mu}(f)(z)\right)^{\prime}}{I_{\lambda}^{p}(a, b, c) L_{\mu}(f)(z)}-\alpha\right)
$$

where the function $p(z)$ is analytic in $\mathcal{U}$ with $p(0)=1$. From the definition of (19), it is easily verified that

$$
z\left(I_{\lambda}^{p}(a, b, c) L_{\mu}(f)(z)\right)^{\prime}=(\mu+1) I_{\lambda}^{p}(a, b, c) f(z)-\mu I_{\lambda}^{p}(a, b, c) L_{\mu}(f)(z) .
$$

Then, by using equations (20) and (21), we obtain

$$
(\mu+1) \frac{I_{\lambda}^{p}(a, b, c) f(z)}{I_{\lambda}^{p}(a, b, c) L_{\mu}(f)(z)}=(1-\alpha) p(z)+\alpha+\mu .
$$

Differentiating (22) we get

$$
\frac{1}{1-\alpha}\left(\frac{z\left(I_{\lambda}^{p}(a, b, c) f(z)\right)^{\prime}}{I_{\lambda}^{p}(a, b, c) f(z)}-\alpha\right)=p(z)+\frac{z p^{\prime}(z)}{(1-\alpha) p(z)+\alpha+\mu}(z \in \mathcal{U}) .
$$

Hence by Lemma (1.1), we conclude that $p(z) \prec \phi(z)$ in $\mathcal{U}$ for $\max _{z \in \mathcal{U}} \operatorname{Re}\{\phi(z)\}<\frac{\alpha+\mu}{\alpha-1}$

which implies that $L_{\mu}(f)(z) \in \mathcal{S}_{\lambda, p}^{*}(\alpha: a, b, c: \phi)$.

Theorem 3.2 Let $\phi \in \mathcal{N}$ with $\max _{z \in \mathcal{U}} \operatorname{Re}\{\phi(z)\}<\frac{\alpha+\mu}{\alpha-1}(c>-1 ; 0 \leq \alpha<1)$. If $f \in \mathcal{K}_{\lambda, p}(\alpha: a, b, c: \phi)$, so is $L_{\mu}(f)$. 
Proof. By applying Theorem(3.1), it follows that

$$
\begin{aligned}
f(z) \in \mathcal{K}_{\lambda, p}(\alpha: a, b, c: \phi) & \Longleftrightarrow z f^{\prime}(z) \in \mathcal{S}_{\lambda, p}^{*}(\alpha: a, b, c: \phi) \\
& \Longleftrightarrow F_{\mu}\left(z f^{\prime}(z)\right) \in \mathcal{S}_{\lambda, p}^{*}(\alpha: a, b, c: \phi) \\
& \Longleftrightarrow z\left(F_{\mu}(f)(z)\right)^{\prime} \in \mathcal{S}_{\lambda, p}^{*}(\alpha: a, b, c: \phi) \\
& \Longleftrightarrow F_{\mu}(f)(z) \in \mathcal{S}_{\lambda, p}^{*}(\alpha: a, b, c: \phi)
\end{aligned}
$$

which proves Theorem(3.2).

Theorem 3.3 Let $\phi, \psi \in \mathcal{N}$ with $\max _{z \in \mathcal{U}}(\operatorname{Re}\{\phi(z)\})<\frac{\mu+\sigma}{\sigma-1}(c>0 ; 0 \leq \sigma<1)$ If $f \in \mathcal{C}_{\lambda, p}^{\prime}(\alpha, \beta ; a, b, c ; \phi, \psi)$, then $F_{\mu}(f) \in \mathcal{C}_{\lambda, p}^{\prime}(\alpha, \beta ; a, b, c ; \phi, \psi)$.

Proof. Let $f(z) \in \mathcal{C}_{\lambda, p}^{\prime}(\alpha, \beta ; a, b, c ; \phi, \psi)$. Then, in view of the definition of the function class $\mathcal{C}_{\lambda, p}^{\prime}(\alpha, \beta ; a, b, c ; \phi, \psi)$, there exists a function $g \in \mathcal{K}_{\lambda, p}(\alpha$ : $a, b, c: \phi)$ such that

$$
\frac{1}{1-\beta}\left(\frac{z\left(I_{\lambda}^{p}(a, b, c) f(z)\right)^{\prime}}{I_{\lambda}^{p}(a, b, c) g(z)}-\beta\right) \prec \psi(z)(z \in \mathcal{U}) .
$$

We set,

$$
p(z)=\frac{1}{1-\beta}\left(\frac{z\left(I_{\lambda}^{p}(a, b, c) F_{\mu}(f)(z)\right)^{\prime}}{I_{\lambda}^{p}(a, b, c) F_{\mu}(g)(z)}-\beta\right)
$$

where the function $p(z)$ is analytic in $\mathcal{U}$ with $p(0)=1$. Using equation (8), we find

$$
\text { that } \begin{aligned}
\frac{1}{1-\beta} & \left(\frac{z\left(I_{\lambda}^{p}(a, b, c) f(z)\right)^{\prime}}{I_{\lambda}^{p}(a, b, c) g(z)}-\beta\right) \\
\quad= & \frac{1}{1-\beta}\left(\frac{z\left(I_{\lambda}^{p}(a, b, c) z f^{\prime}(z)\right)}{I_{\lambda}^{p}(a, b, c) g(z)}-\beta\right) \\
\quad= & \frac{1}{1-\beta}\left(\frac{z\left(I_{\lambda}^{p}(a, b, c) L_{\mu}\left(z f^{\prime}\right)(z)\right)^{\prime}+\mu I_{\lambda}^{p}(a, b, c) L_{\mu}(f)(z)}{z\left(I_{\lambda}^{p}(a, b, c) L_{\mu}(g)(z)\right)^{\prime}+\mu I_{\lambda}^{p}(a, b, c) L_{\mu}(g)(z)}-\beta\right) .
\end{aligned}
$$

Then, by using the same techniques as in the proof of Theorem(2.5), we conclude that

$$
\frac{1}{1-\beta}\left(\frac{z\left(I_{\lambda}^{p}(a, b, c) f(z)\right)^{\prime}}{I_{\lambda}^{p}(a, b, c) g(z)}-\beta\right)=p(z)+\frac{z p^{\prime}(z)}{(1-\sigma) r(z)+\sigma+\mu} \prec \psi(z) .
$$

Hence, upon setting $w(z)=\frac{1}{(1-\sigma) r(z)+\sigma+\mu}$ in equation (24), applying Lemma(1.2), we find that $p \prec \psi$ in $\mathcal{U}$ which yields $F_{\mu}(f) \in \mathcal{C}_{\lambda, p}^{\prime}(\alpha, \beta ; a, b, c ; \phi, \psi)$.

Using a similar argument as in Theorem(3.2), we can prove

Theorem 3.4 Let $\phi, \psi \in \mathcal{N}$ with $\max _{z \in \mathcal{U}}(\operatorname{Re}\{\phi(z)\})<\frac{\mu+\sigma}{\sigma-1}(c>0 ; 0 \leq \sigma<1)$. If $f \in \mathcal{U}_{\lambda, p}^{*}(\alpha, \beta ; a, b, c ; \phi, \psi)$, then $L_{\mu}(f) \in \mathcal{U}_{\lambda, p}^{*}(\alpha, \beta ; a, b, c ; \phi, \psi)$. 


\section{References}

[1] P.L. Duren, Univalent functions, Springer - verlag, Berlin, 1983.

[2] A.W. Goodman, Univalent functions, Vol I, II, Mariner, Tampa, FL, 1983.

[3] I.S. Jack, Functions starlike and convex of order $\alpha$, J. London Math. Soc. 2, 3(1971), 469-474.

[4] Jin-Lin Liu, Some Applications of Certain Integral Operator,Kyungpook Math.J., 43(2003), 211-219.

[5] I.B. Jung, Y.C. Kim and H.M. Srivastava,The Hardy space of analytic functions associated with certain one-parameter families of integral operators, J. Math. Anal. Appl., 176(1993), 138-147.

[6] S. S. Miller, P. T. Mocanu, Differential subordinations and inequalities in the complex plane, J. Differ. Equations, 67 (1987), 199-211.

[7] S. S. Miller, P. T. Mocanu, Differential subordinations and univalent functions, Michigan Mathematical Journal, 28 (1981), 1157-171.

[8] K.I. Noor, On alpha- quasi-convex functions and related topics, Inter. J. Math. and Math. Sci. 10(2), (1987), 241-258.

[9] K.I. Noor: On new classes of integral operators, J. Nat. Geomet. 16, (1999), 71-80.

[10] K.I. Noor, M.A. Noor, On integral operators, J. Math. Anal. Appl. 238, (1999), 341-352.

[11] C.Selvaraj, A subclass of close-to-convex functions, Southeast Asian Bull. of Math., 28, (2004), 113-123.

Received: June 15, 2007 